\title{
Creativity and Delusions: The Dopaminergic Modulation of Cortical Maps
}

\author{
Luís Alfredo Vidal de Carvalho ${ }^{1,3}$, Daniele Quintella Mendes ${ }^{1}$, and \\ Roseli S. Wedemann ${ }^{2,3}$ \\ 1 COPPE - Universidade Federal do Rio de Janeiro, \\ Programa de Engenharia de Sistemas e Computação, \\ P.O. Box 68511, 21941-972, Rio de Janeiro, RJ, Brazil \\ LuisAlfredo@ufrj.br, daniele@cos.ufrj.br \\ 2 Instituto de Matemática e Estatística, Universidade do Estado do Rio de Janeiro \\ Rua São Francisco Xavier, 524, 20550-013, Rio de Janeiro, RJ, Brazil \\ roseli@ime.uerj.br \\ 3 Laboratório UniCarioca de Pesquisa Aplicada, UniCarioca \\ Av. Paulo de Frontin 568, 20261-243, Rio de Janeiro, RJ, Brazil
}

\begin{abstract}
Since little is still known about fundamental brain mechanisms associated to thought, its different manifestations are usually classified in an oversimplified way into normal and abnormal, like delusional and disorganized thought or creative thinking. Considering dopaminergic signal-to-noise neuronal modulation in the central nervous system, and the existence of semantic maps in the human brain, we developed a selforganizing neural network model to unify different thought processes into a single neurocomputational substrate. We performed simulations varying dopaminergic modulation and observed the total patterns that emerged at the resulting semantic map, assuming that these correspond to thought. The model thus shows how normal and abnormal thinking are generated, and that there are no clear borders between their different manifestations. Actually, a continuum of different qualitative reasoning, ranging from delusion to disorganized thought, and passing through normal and creative thinking, seems to be more plausible.
\end{abstract}

\section{Introduction}

One of the most interesting and fuzzy of our mental activities is referred to as creativity. Many have tried to define and partially explain the creative phenomenon. It could be the combination of ideas from different and largely separate knowledge fields, or the ability of making unusual relationships or unexpected connections between elements [1].

Focusing attention on the central elements of a problem and disregarding the peripheral ones is a good strategy for finding a conventional and unique solution to a problem. However, broadening attention to a wider range of elements and regarding them as potentially relevant may be a better approach to finding new and creative solutions. This divergent thought style follows many directions in parallel and allows the discovery of unusual associations of ideas. 
Psychodynamical theories were also proposed to explain creativity. Freud suggested that the creative act is a consequence of a fantastic view of the world, when reality frustrates a subject's desires. If incursion to fantasy does not alleviate excessive frustration, the subject may develop neurotic or psychotic symptoms, which are pathological disguises for infantile fantasies [2].

Although inconclusive [2,3], psychodynamical theories gather in a single model creativity, psychopathology and unconsciousness. Indeed, many reports express a strong correlation between creative and psychotic thinking. In the seventies, creative writers and maniacs were compared and a common tendency to broaden or shift conceptual boundaries (overinclusion) was observed [4]. In the eighties, creativity and schizophrenic thought were suggested to be related to the same cognitive process, based on the Alternate Uses Tests [5]. Whatever the relation between psychopathology and creativity is, they seem to have some common aspects, like the idea of broader, distant or looser association making and unfocusing of attention. In the present paper, we explore these commonalities to define an unifying model for creative and disturbed thought.

As described by Karl Jaspers [6], delusions are thought processes that deviate from normal logical thinking because they are manifest by ideas with characteristics such as subjective certainty, incorrigibility, and impossibility of content. Delusions cannot be understood and corrected even in the presence of many logical arguments. This classical characterization of delusional symptoms has been reviewed, since for example, some delusions are indeed possibly true and many of these characteristics can be applied to religious convictions. Impossible, improbable, or even true, a delusion is a statement made in an inappropriate context or without a logical justification based on present reality. Delusions are not followed by adequate and reasonable justifications and their property of total and unquestionable certainty leads to their incorrigibility.

For Freud, a delusion is a defense process where judgment mistakes are made, when the ego tries to isolate from consciousness intolerable representations. When an intolerable idea is inseparably connected to reality, the only way of isolating it from consciousness is to detach from reality. Delusions are stimulated by a mixture of anxiety, hiperarousal, suspicion, and the attachment of meaning to insignificant events.

Disorganized thought is characterized by a loss of the capacity to associate ideas in a logical way. Ideas completely heterogeneous to each other are associated, so that the subject's discourse becomes incoherent, and many times unintelligible. This phenomenon is observed specially in schizophrenia, but also in delirium and in excited maniac patients. In schizophrenia, disorganized thought, along with delusions and hallucinations, is considered a positive psychotic symptom, and responds well to neuroleptic treatment.

The remainder of the paper is organized as follows. We will describe the dopaminergic hypothesis, on which our model is based, in the next section. In section 3, we present our neural network model of cortical map formation of mental representations. In the final section, we describe the simulation experiments and their results, and draw our conclusions. 


\section{Dopaminergic Modulation}

The catecholamines norepinephrine, epinephrine, and dopamine are important neuroactive substances produced in some brain sites and released at distant and widespread areas in a diffuse or divergent way. These neuromodulating substances do not act through membrane ion channels but, instead, activate intracellular messengers, promoting a longer effect than the other neurochemicals released by synapses inside the brain. Since these other chemicals, called neurotransmitters, have specific and local synaptic patterns, act through ion channels, and have short-lasting effects, it is interesting to suppose that they differ from the catecolamines in function. Due to the fast action and connection patterns of their producing synapses, neurotransmitters seem to be involved in the immediate processing of signals, while the neuromodulators, with their opposing properties, suggest a regulatory function, modulating the operational characteristics of the receptor neurons, i.e., their responses to neurotransmitters.

Increases or decreases in the catecholaminergic levels have behavioral consequences in arousal, attention, learning, memory, and motor responses [7]. It is still not clearly verified, but it seems plausible to assume that catecholamines affect the neuronal ability to discern what is information from what is noise in a signal. Some authors suggest that these neuromodulators enhance the stronger signal and dampen the weak one [8], while others advocate that they enhance the cell sensitivity to either excitatory or inhibitory signals [9]. Whatever the mechanism is, the net effect is the enhancement of the signal in relation to the background, spontaneous activity called "noise".

The dopamine hypothesis of schizophrenia advocates that the disorder is caused by an overactivity of the brain dopaminergic system [8]. An elaboration of this hypothesis is that the dopamine release is chronically reduced in schizophrenic patients, leading to the upregulation of the postsynaptic receptors and a consequent intensified response in moments of normal or increased dopamine release, for example, due to environmental stressors [10]. This would explain both, the positive and the negative, symptoms of the disease.

A relation between acute delusions and dopamine activity is clear from the fact that amphetamine can cause psychotic states with paranoia, hyperarousal, hyperactivity, and suspiciousness. It also seems that a decreased dopamine level leads to a lower signal-to-noise ratio and looser associations of thought, allowing the creation of new relations [8].

\section{Cortical Maps}

Since mid 19th century, models and experimental results have shown that brain cells in many animals have a structure called on-center/off-surround, in which a neuron is in cooperation, through excitatory synapses, with other neurons in its immediate neighborhood, while it is in competition with other neurons that lay outside these surroundings [11]. 
Competition and cooperation are found not only statically hardwired, but also as part of many neuronal dynamical processes. As a matter of fact, competition is essential to neurodevelopment where neurons compete for certain chemicals. In synaptogenesis, for example, substances generically called neural growth factors are released by stimulated neurons and, spreading through diffusion, reach the neighboring cells, promoting synaptic growth. Cells that receive neural growth factors make synapses and live, while cells that have no contact with these substances die [12]. A neuron that releases neural growth factor guides the process of synaptic formation in its tri-dimensional neighborhood, becoming a center of synaptic convergence. When some neighboring neurons release different neural growth factors in different amounts, many synaptic convergence centers are generated and a competition is established between them through the synapses of their surroundings. It seems that at least two processes participate in the dynamics of synaptic formation: pre-synaptic neurons competing for neural growth factors to survive, and post-synaptic neurons that release neural growth factors competing for synapses that will keep them alive with stimuli. A signaling network is thus established to control the development and plasticity of neuronal circuits. Remembering that all this competition is started and controlled by environmental stimulation, it is possible to have an idea of the way the environment records or represents itself in the brain.

The competition processes described above are essential to the formation of some neuronal organizations called maps. A neural map is a biological circuit composed of two sets of neurons, called domain and image, in such a way that similar patterns of activation of the domain are projected to neighboring neurons in the image. These maps are subject to constant change, not only in the neurodevelopmental phase, but throughout life as a function of the subject's experiences.

Maps have puzzled neuroscientists in the last decades, mainly the question of how they arise from the simple on-center/off-surround wiring pattern. Computational theories gave some important insights to the problem, since some cortical maps can be artificially developed from simple governing rules for synaptic plasticity, in computer simulation models. The most general of these models is called the Self-Organizing Map [13], in which two sheets of neuronal tissue with $n$ neurons each, corresponding to the domain and the image, are initially randomly connected in a way that every neuron $i$ at the image receives synaptic projections $w_{i} \in \Re^{n}$ from every neuron at the domain (see Figure 1). Neurons at the domain don't form synapses among themselves and receive "sensory" inputs (stimuli), while neurons at the image make synapses following the on-center/off-surround paradigm, i.e., short-range excitation or cooperation and long-range inhibition or competition (see Figure 2).

The on-center/off-surround synapses don't change during the development of the map, while the synapses between the domain and the image are modified along the process of map formation. Indeed, every time the neural network is in contact with a stimulus $x_{k} \in \Re^{n}, k=1,2, \ldots$ in its domain, there will be only one excited neuron $i^{*}$ at the image because the short-range cooperation 


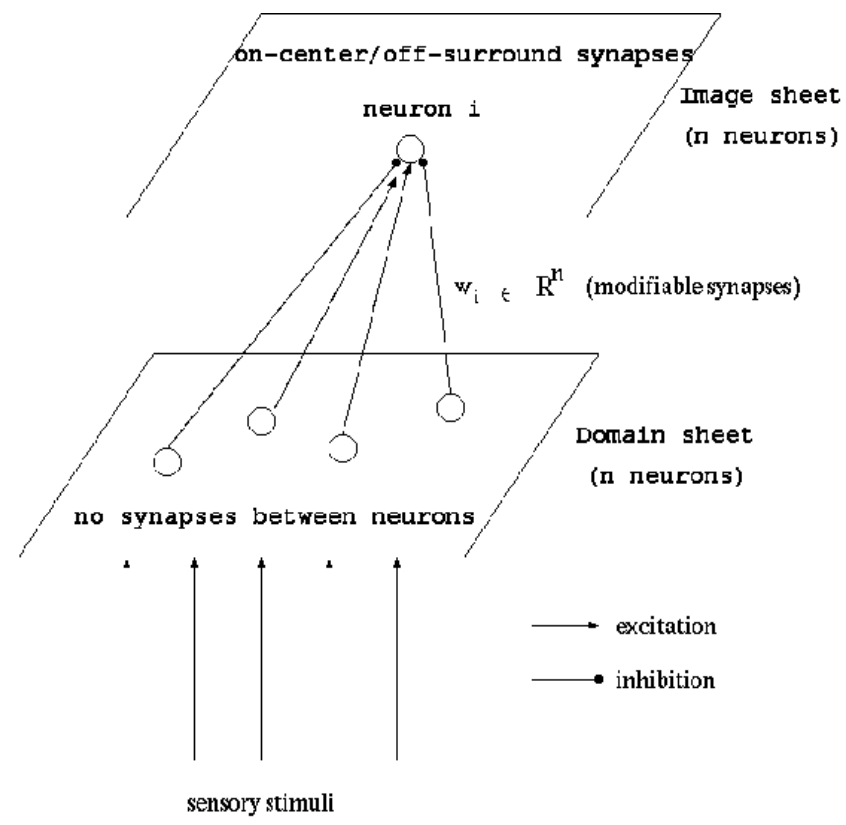

Fig. 1. A Self organizing map with two bi-dimensional sheets of neurons.

and long-range competition makes the more excited neuron inhibit the others. The position $r^{*}$ of this winner neuron at the image determines how much the synapses will be modified. Synapses from neurons closer to the winner will be strongly changed, in such a way that these neurons will be more intensely excited by the stimulus $x_{k}$ in the future. Synapses from neurons distant from the winner will be weakly changed or not changed at all, depending on the dispersion $\sigma$ of the neighborhood function $\phi\left(r_{i}, r^{*}\right)$, where $r_{i} \in \Re^{n}$ gives the position of a neuron $i$ at the image sheet. By this process, every neuron in the image will be more easily excited by the stimulus $x_{k}$ (synaptic facilitation) in the future. The development of the map is a consequence of the fact that the amount of synaptic facilitation is a function of the distance from the winner neuron. The process of synaptic modification represented by $\Delta w_{i}^{l}$ for each neuron $i$ is repeated for every learning step $l$, where the stimulus $x_{k} \in \Re^{n}, k=1,2, \ldots$ is presented to the neural network, and is given by

$$
\Delta w_{i}^{l}=\rho(l) * \phi\left(r_{i}, r^{*}\right) *\left(x_{k}-w_{i}\right),
$$

where $\rho(l)$ is the learning rate defined by

$$
\rho(l)=\rho_{0} * \beta^{(l-1)}, 0<\beta<1, l=1,2, \ldots
$$

The learning rate begins with the value $\rho_{0}$ and decreases at each learning step $l$ with a rate $\beta$. The neighborhood symmetric function $\phi\left(r_{i}, r^{*}\right)$ takes the 


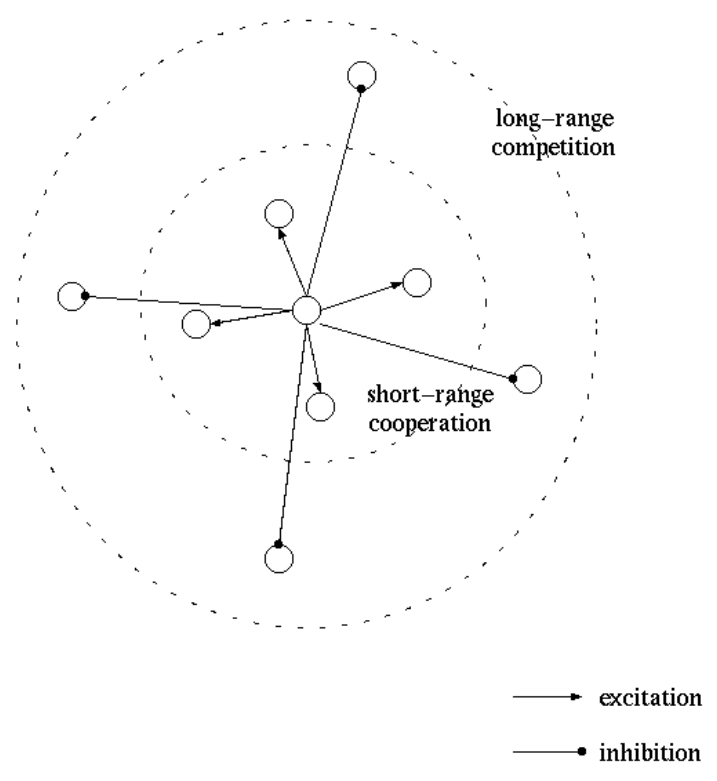

Fig. 2. The On-center/Off-surround synaptical pattern of the image neuronal sheet.

form of a gaussian function

$$
\phi\left(r_{i}, r^{*}\right)=\exp -\left(\frac{\left|r_{i}-r^{*}\right|^{2}}{2 \sigma(l)^{2}}\right),
$$

where the dispersion $\sigma(l)$ at each learning step is given by

$$
\sigma(l)=\sigma_{0} * \alpha^{(l-1)}, 0<\alpha<1, l=1,2, \ldots,
$$

and $\alpha$ is a decrement rate.

The initial dispersion of the gaussian, $\sigma_{0}$, is high, representing that all the neurons in the image are considered neighbors. This allows the modification of the randomness of the initial synapses to a more organized pattern, where neighborhood is of capital importance. At every time-step $l$ in which another stimulus is presented to the neural network domain, the neighborhood shrinks a bit, gradually giving the map a local organization.

\section{Simulation Results and Conclusions}

A self-organizing neural network with its two bi-dimensional sheets composed of 400 neurons each was developed for computer simulation, as shown in Figure 1. A set of different stimuli, symbolized by geometrical markers and representing different concepts or ideas, was repeatedly presented to the Domain sheet of the 
neural network. Due to the feedforward connections between the Domain and the Image sheet, every stimulus presented to the Domain is projected to the Image. Initially, synapses are randomly generated and therefore the stimuli presented to the Domain sheet are projected to random positions at the Image layer. While the stimuli are repeatedly presented to the neural network, synapses change and a map-like structure develops at the Image layer. Similar stimuli, representing nearly associated or similar concepts, when presented to the Domain layer, lead to the excitation of neighboring regions in the Image neuronal layer. This is what we call a semantic map.

The purpose of our simulations is to show that different maps arise when dopaminergic modulation controls the synaptic formation process. In fact, varying the parameters responsible for the signal-to-noise ratio results in maps that represent the concepts or ideas in a way that can be likened to the creative, and disorganized thought. To simulate the signal enhancement promoted by the dopaminergic activity, a threshold $\theta$ is associated to every neuron at the Image sheet [9]. When the total signal input, coming from the Domain layer to an Image sheet neuron, exceeds the threshold, this neuron is considered to be excited. Increasing or decreasing the threshold will promote the effect of dopaminergic dampening or enhancement of the incoming signal. The simulation of noise is simply obtained by adding to the stimulus a random number in the interval $[-p,+p]$, where $p$ is a percentage of the stimulus value [9]. The parameters $\theta$ and $p$ allow us to realize any desirable simulation, with total control flexibility over the signal-to-noise ratio.

In a first simulation experiment, a semantic map was allowed to develop from the self-organizing neural network when ten stimuli, representing ten different concepts or ideas, were repeatedly presented to the Domain layer with no noise and a predefined signal level $\theta$ of 0.999 . This map is represented in Figure 3.

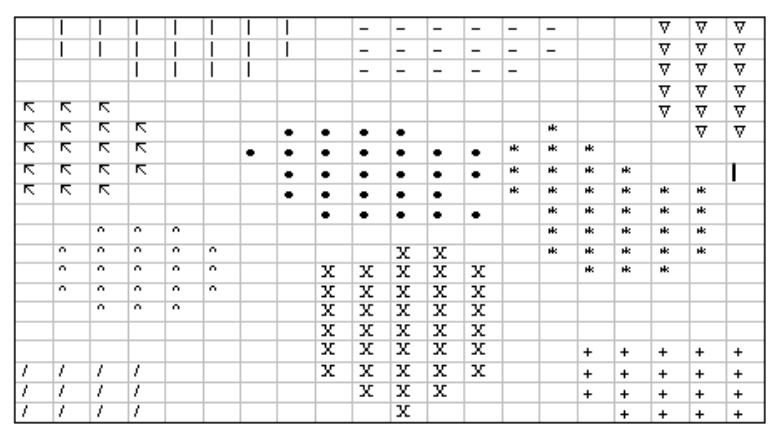

Fig. 3. A reference map with 10 different concepts represented on it.

In a second simulation, the Domain sheet of this already well-formed map was excited by the single stimuli represented with a $\bullet$. The dopaminergic modulation was changed in this simulation with the addition of a noise level $p$ of $10 \%$. The 
resulting Image layer map can be seen in Figure 4. Note that with the addition of noise, the stimulus • expanded its representation, exciting neurons outside its original region at the Image layer and invading the region associated to the concept represented by $*$. This can be interpreted as if the increase of noise level, or equivalently the decrement of the signal-to-noise ratio, was capable of promoting the association of the different, but similar, ideas or concepts $\bullet$ and *, neighbors in the map.

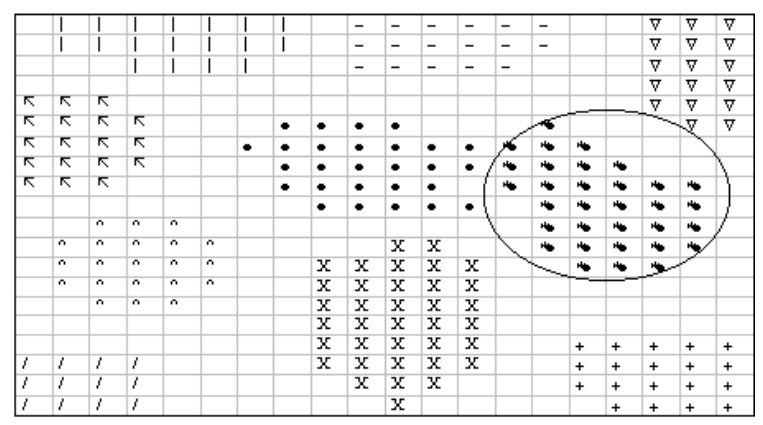

Fig. 4. The central idea $\bullet$ (thesis) is associated with a neighboring idea $*$ (antithesis), leading to the formation of a pattern that is the conclusion of the thinking process, or the synthesis.

Much of our reasoning about the functioning of our model can be understood as a mechanism of association of ideas. Indeed, when a stimulus (endogenous or exogenous) elicits a central idea, that we will call here a "thesis", other ideas, that corroborate or refute the thesis, are spontaneously elicited. Let us call these spontaneously elicited ideas the "antitheses". Because the thesis and the antitheses are elicited at the same time, they are temporally associated, and the final result of this simultaneous presence is the weighted sum of their influences, with the emergence of a final pattern that we will call the "synthesis", or the conclusion of the reasoning process. If we assume that "normal" thought is the triggering of a thesis that elicits a group of antitheses which will be weighted (pondered) together to generate a synthesis, then, for the occurrence of "normal" thought, it is necessary to have some level of noise, i.e., a relatively lower dopaminergic modulation of the signal-to-noise ratio.

In the next simulation, the noise level was increased from $10 \%$ to $170 \%$, and the same procedure followed in the second experiment was repeated. The result is shown in Figure 5. Note that now the central stimulus $\bullet$ (thesis) has excited many neurons outside its original representation, invading areas where other stimuli were represented. In our model, this means that a central idea (thesis) has been associated with many other ideas (antitheses) generating a pattern that we can liken to a creative thinking process. The process of making associations between a central stimulus and distant ones resembles the formerly 
reviewed theories of creativity, where concepts like "loosening of associations", "divergent-thought", "ability of making unusual relationships", "a momentary freedom from stereotyped and conventional scenes", "broadening of conceptual boundaries", and some other similar concepts are always present. As a consequence, it is necessary to have a higher level of noise, or equivalently, a lower dopaminergic modulation of the signal-to-noise ratio, for the occurrence of creative or schizophrenic thinking, as experimentally observed in [7].

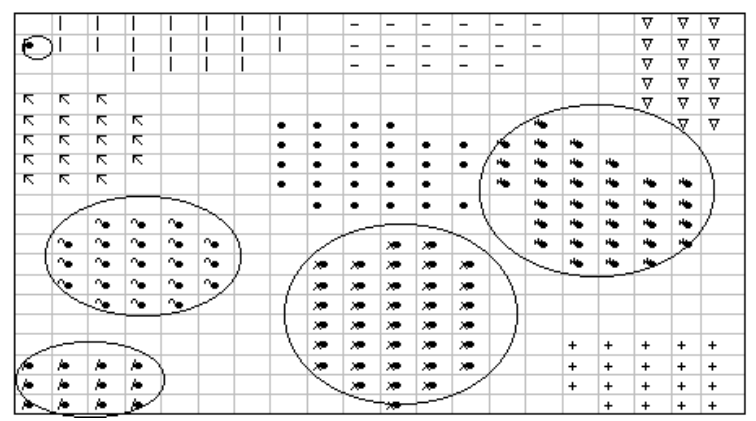

Fig. 5. The central idea $\bullet$ (thesis) is associated with distant ideas (antithesis), leading to the formation of a pattern that can be likened to creative or schizophrenic thinking.

If the signal-to-noise dopaminergic modulation is still more reduced, in the simulation experiences as a consequence of an increase in the noise level $p$, the association of ideas becomes more flexible and the creative thought degenerates to complete disorganization. The border between creativity and disorganization is obviously not clear, as seen in the ideas reviewed at the beginning of this paper. As a consequence, the precise level of dopaminergic modulation of the signal-tonoise ratio that distiguishes geniality from illness can not be determined.

The model unifies the qualitatively different thinking processes into a neurobiologically-based substrate. Different thinking processes are viewed so as to correspond to possible positions over a one-dimensional continuum, where the signal-to-noise ratio is the measure. At one extreme of this line, where the signalto-noise ratio is high, the semantic map becomes more focused in the representations of ideas. At the other end of the linear continuum, where the signal-to-noise ratio is low, the excessive noise promotes unusual associations among ideas, resembling the disorganized thought. The "normal" and the creative thought processes are positioned between these two ends, depending on the noise level, as can be pictorially shown in Figure 6. Although biologically plausible, in agreement with many aspects described by psychodynamical clinical experience, and experimentally based on simulations, the model is very schematic and far from explaining the complexities of human thinking. Nevertheless, it seems to be a good metaphorical and unifying view of the many facets of this phenomenon. 


\section{Signal-to-noise ratio}

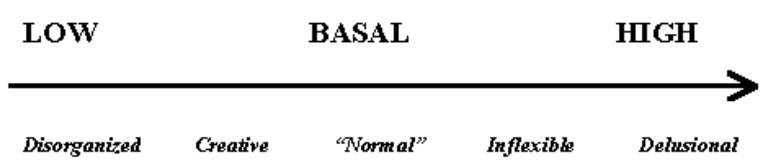

\section{Thought Processes}

Fig. 6. The linear unifying continuum of thought processes, based on the signal-to-noise dopaminergic modulation.

This research was developed with grants from the brazilian National Research Council ( $\mathrm{CNPq})$ and from the Rio de Janeiro State Research Foundation (FAPERJ).

\section{References}

1. Rogers, C.: Towards a Theory of Creativity. In: Anderson, H. (ed): Creativity and its Cultivation. Harper \& Brothers Publishers, New York, (1949).

2. Freud, S.: Introductory Lectures on Psycho-Analysis. Standard Edition, W. W. Norton and Company (1966, first German edition in 1917),

3. Freud, S.: Psycho-analytic Notes on an Autobiographical Account of a case of Paranoia (Dementia Paranoides). In: the Standard Edition of the Complete Psychological Works of Sigmund Freud, Vol. XII. Vintage, The Hogarth Press and the Institute of Psycho-analysis (2001, first German edition in 1911).

4. Andreasen, N. and Powers, P.: Creativity and Psychosis. Archives of General Psychiatry, Vol. 32, (1976) 70-73.

5. Keefe, J. and Magaro, P.: Creativity and Schizophrenia: An Equivalence of Cognitive Processing. Journal of Abnormal Psychology, Vol. 89, n. 3, (1980) 390-398.

6. Jaspers, K.: Psicopatologia Geral. Editora Beta, Buenos Aires, (1966).

7. Spitzer, M.: A Cognitive Neuroscience View of Schizophrenic Thought Disorder. Schizophrenia Bulletin, Vol. 23, n. 1, (1997) 29-50.

8. Spitzer, M.: . A Neurocomputational Approach to Delusions. Comprehensive Psychology, Vol. 36, n. 83, (1995) 83-105.

9. Servan-Schreiber, D., Printz, H. and Cohen, J.: A Network Model of Catecholamine Effects: Gain, Signal-to-Noise Ratio, and Behavior. Science, Vol. 249, (1990) 892895.

10. Grace, A.: Phasic Versus Tonic Dopamine Release and the Modulation of Dopamine System Responsivity: A Hypothesis for the Etiology of Schizophrenia. Neuroscience, Vol. 41, (1991) 1-24.

11. Eccles, J. and Szentagothai, J.: The Cerebellum as a Neuronal Machine. SpringerVerlag, New York, (1967).

12. Kandel, E.: Cellular Mechanisms of Learning and the Biological Basis of Individuality. In: Principles of Neuroscience. Norwalk, Appleton and Lange (1991).

13. Kohonen, T.: Self-Organized Formation of Topologically Correct Feature Maps. Biological Cybernetics, Vol. 43, (1982) 59-69. 